\title{
Initial blood pressure is important for long-term outcome after traumatic spinal cord injury
}

\author{
Mette Haldrup, MD, ${ }^{1}$ Stig Dyrskog, MD, PhD, ${ }^{2}$ Mathias Møller Thygesen, MD, ${ }^{1}$ \\ Hans Kirkegaard, MD, DMSc, PhD, DEAA, DLS, ${ }^{3}$ Helge Kasch, MD, PhD, ${ }^{4}$ and \\ Mikkel Mylius Rasmussen, MD, PhD'
}

\begin{abstract}
'Department of Neurosurgery, CENSE-Spine, Aarhus University Hospital; ${ }^{2}$ Department of Neuro-Intensive Care, Aarhus University Hospital; ${ }^{3}$ Department of Prehospial Care, Region Midt; and ${ }^{4}$ Department of Clinical Medicine, Spinal Cord Injury Center of Western Denmark, Aarhus, Denmark
\end{abstract}

\begin{abstract}
OBJECTIVE Patients with traumatic spinal cord injury (TSCl) are at risk of developing neurogenic shock that causes hypotension and thereby secondary injury to the spinal cord due to ischemia. Hemodynamic treatment of patients with acute TSCl remains inadequately elucidated. Guidelines for management are divergent and based on limited evidence. To this end, the authors evaluated whether mean arterial blood pressure (MABP) during the prehospital and initial hospital phases of TSCl treatment is correlated with long-term neurological outcome.
\end{abstract}

METHODS The authors performed a retrospective cohort study based on a chart review of MABP data collected during the prehospital transport, in the operating room (OR), and in the neurointensive care unit (NICU) during the first 7 days after trauma. Data from the NICU were divided into two periods: days 1-2 and days 3-7. Data were analyzed using Spearman's rank correlation to evaluate for any correlation between MABP and changes in the International Standards for Neurological Classification of Spinal Cord Injury (ISNCSCI) score 1 year postinjury. In the analysis, the MABP target value was $80 \mathrm{~mm} \mathrm{Hg}$. Hypotension was treated with metaoxedrin or norepinephrine. Statistically significant differences were evaluated using Spearman's rank correlation coefficient.

RESULTS The chart review yielded 129 patients treated for TSCI. The inclusion period was 2010-2017. For the prehospital transport measurements of MABP, the Spearman's rank correlation coefficient was a rho of $0.5662(p<0.001)$, for OR measurements it was a rho of $0.6818(p<0.001)$, and for the NICU measurements it was a rho of $0.4611(p<0.001)$; for NICU unit days 1-2 and days 3-7, the Spearman's rank correlation coefficient was a rho of $0.2209(p=0.0681)$.

CONCLUSIONS Continuous MABP levels exceeding $80 \mathrm{~mm} \mathrm{Hg}$ have a significant impact on neurological outcomefrom earliest possible stabilization in the prehospital care, through hospital admission, the surgical phase, and into the first 2 days in the NICU.

https://thejns.org/doi/abs/10.3171/2020.1.SPINE191005

KEYWORDS traumatic spinal cord injury; mean arterial blood pressure; prehospital; operating room; neurointensive care unit; neurological outcome; trauma

$\mathrm{T}$ HE annual worldwide incidence of spinal cord injury (SCI) ranges from 20 to 50 injuries per million persons. Spontaneous recovery depends on the severity of the injury and varies from patient to patient, although the majority of recoveries occur within the first 3 months after the trauma. ${ }^{1}$ The consequences of traumatic SCI are severe. The combined motor and sensory neurological deficits, in addition to the neurogenic bladder, bowel, and sexual dysfunction, have a vast impact on the individual's quality of life, ${ }^{2,3}$ with a close correlation to the completeness of SCI. ${ }^{4}$

Due to sympathetic denervation, acute traumatic SCI causes bradycardia, peripheral vasodilation, and hypotension, in turn causing neurogenic shock. ${ }^{5,6}$ Spinal cord blood flow has been subject to detailed study in animal models of SCI, and its impairment is believed to contribute to neurological injury after SCI. Patients with SCI frequently experience autonomic and hemodynamic instabil-

ABBREVIATIONS AIS = American Spinal Injury Association Impairment Scale; ISNCSCI = International Standards for Neurological Classification of Spinal Cord Injury; $\mathrm{MABP}=$ mean arterial blood pressure; $\mathrm{NICU}=$ neurointensive care unit; $\mathrm{OR}=$ operating room; $\mathrm{SCl}=$ spinal cord injury.

SUBMITTED August 27, 2019. ACCEPTED January 20, 2020.

INCLUDE WHEN CITING Published online March 20, 2020; DOI: 10.3171/2020.1.SPINE191005. 
ity in the 1st week after injury. ${ }^{8}$ Hypotension leads to lower perfusion pressure in the spinal cord and thus increases the possibility of secondary ischemic injury to the spinal cord. ${ }^{9-12}$ Furthermore, trauma-induced hypovolemia may exacerbate these effects. ${ }^{10}$

The optimal treatment of hypotension in acute traumatic SCI remains to be clarified, and divergent management strategies exist. ${ }^{10,13}$ Overall, the care of patients with acute SCIs include early immobilization of the spinal column, rapid triage, transport to a definitive care unit, and administration of inotropes and crystalloids. The latter is used to prevent secondary injury by preserving mean arterial blood pressure (MABP) and thereby spinal cord tissue..$^{13,14}$

The first guidelines for the management of acute SCI were published in 2002.14,15 They recommended that hypotension, defined as a systolic blood pressure $<90 \mathrm{~mm}$ $\mathrm{Hg}$, should be avoided and that an MABP of $85-90 \mathrm{~mm}$ $\mathrm{Hg}$ should be targeted in the first 7 days after injury. ${ }^{15}$ These recommendations were essentially unchanged in the 2013 update of the guidelines. ${ }^{16}$ Newer studies testing the guidelines, as applied in the first 7 days of hospitalization, found a correlation between a controlled MABP $>85$ $\mathrm{mm} \mathrm{Hg}$ and a better neurological outcome in these cases. ${ }^{6,17,18}$ However, the ideal MABP is still being debated, and practice patterns vary among centers.

Earlier studies on MABP and neurological outcome have included data solely from the neurointensive care unit (NICU). ${ }^{5,13}$ However, secondary ischemic injury may emerge before admission to the NICU. Prehsopital MABP and operating room $(\mathrm{OR}) \mathrm{MABP}$, and their correlation to end-neurological outcome, were not included in earlier studies. These MABP values range over an important time period in the first phase after trauma, and a decrease in blood pressure here might have a detrimental impact on a patient's long-term neurological outcome. At our institution, MABP data collected by the prehospital service are available from the trauma site and during transport to the hospital. We aimed to investigate the correlation between long-term neurological outcome and MABP during transport to the hospital, in the OR, and during the first 7 days of hospitalization in the NICU.

\section{Methods}

This is a cohort study based on data from a retrospective chart review. Patients were identified using the Western Denmark Spinal Cord Injury database. The database contains data on every patient who has undergone rehabilitation after traumatic SCI at the Spinal Cord Injury Centre of Western Denmark (population-based data). We included all patients with traumatic SCI from January 2010 through December 2017. Data from prehospital treatment and transport, from the OR, and from the first 7 days of hospitalization in the NICU were collected from the Prehospital Patient Journal, the Electronic Patient Journal, and older paper files. We also obtained data on neurological outcome at admission to the hospital and 1 year after the time of injury from the Western Denmark Spinal Cord Injury database, from the Electronic Patient Journal, and older paper files. The vast majority of cases were due to sports, falls, or traffic accidents. Hypotension was treated with metaoxedrin or norepinephrine in the NICU if MABP was below $80 \mathrm{~mm} \mathrm{Hg}$. MABP was kept at a threshold for at least 7 days postinjury.

We evaluated neurological outcome using the International Standards for Neurological Classification of Spinal Cord Injury (ISNCSCI) score and the American Spinal Injury Association Impairment Scale (AIS) grade. ${ }^{9}$ AIS grade $\mathrm{A}$ is defined as complete SCI, with no sensory or motor function preserved in the S4-5 segments; grade $\mathrm{B}$ as motor complete injury; grade $\mathrm{C}$ as motor function preserved in more than half of the key muscles below the injury level graded less than 3 (grade 3: active movement and full range of motion against gravity); grade D as motor function preserved in half of the key muscles, with motor function better or equal to 3; and grade $\mathrm{E}$ as normal function in a patient with prior SCI deficits.

Improvement of neurological outcome was defined as a positive decrease in AIS grade. Worsened outcome was defined as a negative increase in AIS grade.

\section{Data Collection and Management}

For every patient we registered 3 sets of MABP values: 1) Prehospital transport data were noninvasively (cuffmeasured) obtained and registered every 15 minutes; 2) OR data were measured invasively (arterial cannula) or noninvasively (cuff-measured) and were registered every 15 minutes; and 3) data from the first 7 days of hospitalization in the NICU were monitored invasively. These data were further separated into two periods: days 1-2 in the NICU and days 3-7 in the NICU, and blood pressure measurements were registered every hour. MABP events were recorded in each group (prehospital, OR, and NICU) and the statistics were done separately for the abovementioned groups that were considered in the acute and more subacute phases of NICU care. If MABP were registered more often, these MABP values were included as well. Groups were defined as follows: Prehospital data were those obtained until patients arrived at the first care-giving hospital. NICU data were those obtained at all times after that, except for when the patient was in the OR. Patients too unstable for decompressive spine surgery, patients who needed treatment outside the NICU (i.e., due to other trauma), or patients who required other procedures before undergoing spine surgery were not included in the project.

Until 2018, the guideline at our center for an MABP threshold was $>80 \mathrm{~mm} \mathrm{Hg}$. Therefore, we divided the collected MABP values into two categories: MABP $<80$ $\mathrm{mm} \mathrm{Hg}$ and $\mathrm{MABP} \geq 80 \mathrm{~mm} \mathrm{Hg}$. For every patient, we counted the number of events in each category. Because the number of MABP events that were registered varied for most patients, especially because of variations in transport time and OR time, the number of events of MABP being $<80 \mathrm{~mm} \mathrm{Hg}$ was computed to a relative value for all events for prehospital data, OR data, and NICU data. In order to evaluate whether these were consequences of having events of blood pressure $<80 \mathrm{~mm} \mathrm{Hg}$, patients were classified into the following: those with no events of MABP $<80 \mathrm{~mm} \mathrm{Hg}$; those with $0.1 \%-33.2 \%$ of events of MABP $<80 \mathrm{~mm} \mathrm{Hg}$; those with $33.3 \%-49.9 \%$ of events of MABP $<80 \mathrm{~mm} \mathrm{Hg}$; and those with $50 \%-100 \%$ of events of $\mathrm{MABP}<80 \mathrm{~mm} \mathrm{Hg}$. 
TABLE 1. Summary of patient demographics

\begin{tabular}{lc}
\hline \multicolumn{1}{c}{ Patient Demographics } & Value \\
\hline Male/female & $105: 24$ \\
\hline Mean age at trauma (range), yrs & $50(20-80)$ \\
\hline
\end{tabular}

In order to evaluate the patients' outcome, we used their AIS grade at admission and at 1 year postinjury. Patients were divided into 3 groups: improved-all patients with improved AIS grade (i.e., A to B or better, B to C or better, etc.) after 1 year; unchanged-all patients with unchanged AIS grade after 1 year; and worsened-all patients with worsened AIS grade (i.e., D to C or worse, C to B or worse, etc.) after 1 year.

\section{Statistical Analysis}

Spearman's rank correlation was used to evaluate correlation between MABP and change in AIS grade 1 year posttrauma. Statistically significant differences were evaluated by Spearman's rank correlation coefficient (range -1 to 1 ) and matching $p$ value. The coefficient, positive or negative, describes the strength of a correlation ( $\rho$ of $0.00-0.19$, for example, indicates very weak correlation; $\rho$ of $0.20-0.39$ indicates a weak correlation; $\rho$ of $0.40-0.69$ indicates a moderate correlation; $\rho$ of $0.70-0.89$ indicates a strong correlation; $\rho$ of $0.90-1.00$ indicates a very strong correlation). Fischer's exact test was applied in order to evaluate possible differences in the neurological outcome between patients with cervical SCI and those with thoracic SCI.

\section{Results}

We included 195 patients (Tables 1 and 2). Sixty-six patients were excluded due to missing MABP data from the prehospital transport, the OR, and/or the NICU. Patients were also excluded if they had a Glasgow Coma Scale score of 3 and had been intubated at the trauma site, making it impossible to establish an AIS grade. This led to enrollment of 129 patients. For all patients, MABP values were collected during the 3 established periods. One hundred fourteen patients had a registered AIS grade at the time of admission to the NICU. For the remaining 15 patients, we reconstructed their AIS grade from their files within the 1st day of admission (Table 3).

We collected data during the prehospital transport for 105 of 129 patients. Among these, 34 patients showed improvement, in 66 patients the condition was unchanged, and in 5 patients the condition had worsened $(\rho=0.5662$, $\mathrm{p}<0.001)$. This underscores a significant correlation between MABP and change in ISNCSCI score at 1 year postinjury with a moderate impact (Fig. 1). We collected data from the OR for 101 of 129 patients. Among these, 30 patients showed improvement, in 68 patients the condition was unchanged, and in 3 patients the condition had worsened $(\rho=0.6818, p<0.001)$, indicating a moderate impact (Fig. 1).

For the first 2 days of hospitalization in the NICU, we collected data from 90 of 129 patients. Among these, 21
TABLE 2. Summary of data pertaining to injury

\begin{tabular}{ccc}
\hline Variable & At Admission & 1 Year Postinjury \\
\hline ISNCSCI score & & \\
\hline AIS grade A & $58(45 \%)$ & $45(35 \%)$ \\
\hline AIS grade B & $10(8 \%)$ & $5(4 \%)$ \\
\hline AIS grade C & $24(19 \%)$ & $27(21 \%)$ \\
\hline AIS grade D & $37(29 \%)$ & $51(40 \%)$ \\
\hline AIS grade E & $0(0 \%)$ & $1(1 \%)$ \\
\hline Improved AIS & $35(27 \%)$ & \\
\hline Unchanged AIS & $89(69 \%)$ & \\
\hline Worsened AIS & $5(4 \%)$ & \\
\hline Level of trauma & & \\
\hline Cervical & $50(39 \%)$ & \\
\hline Thoracic & $46(36 \%)$ & \\
\hline Lumbar & $33(25 \%)$ & \\
\hline
\end{tabular}

Values are presented as the number (\%) of patients.

patients showed improvement in their ISNCSCI score, in 47 patients the condition was unchanged, and in 2 patients the condition had worsened $(\rho=0.4611, p=0.001)$, indicating a moderate impact (Fig. 1). For the remaining days 3-7 in the NICU, data were available for 69 of 129 patients. Among these, 21 patients showed improvement in ISNCSCI score, in 46 patients the condition was unchanged, and in 2 patients the condition had worsened ( $\rho$ $=0.2209, p=0.0681)$. What these results show is in contrast to the remaining data, indicating no correlation between MABP for the last days in the NICU and change in ISNCSCI score (Fig. 1). No difference was found in neurological outcome between cervical and thoracic injured patients $(\mathrm{p}=0.29)$.

\section{Discussion}

We found a moderate but significant correlation between an MABP threshold of $80 \mathrm{~mm} \mathrm{Hg}$ and long-term neurological outcome from the prehospital period, through surgery and into the first critical time in the NICU. Patients with an MABP $\geq 80 \mathrm{~mm} \mathrm{Hg}$ with none or only few events (MABP $<80 \mathrm{~mm} \mathrm{Hg}$ ) had significantly better ISNCSCI score 1 year post-SCI.

Earlier studies evaluating the impact of MABP during hospitalization in the NICU found a correlation between a well-regulated MABP (> $80 \mathrm{~mm} \mathrm{Hg})$ and an improved

TABLE 3. Changes in AIS grade

\begin{tabular}{crrrrr}
\hline AIS Grade at & \multicolumn{5}{c}{ AIS Grade 1 Year Postinjury } \\
\cline { 2 - 6 } Admission & A & B & C & D & E \\
\hline A & 42 & 3 & 8 & 3 & 0 \\
\hline B & 1 & 2 & 5 & 3 & 0 \\
\hline C & 0 & 1 & 12 & 11 & 0 \\
\hline D & 1 & 0 & 2 & 35 & 1 \\
\hline
\end{tabular}

Values are presented as the number of patients. 

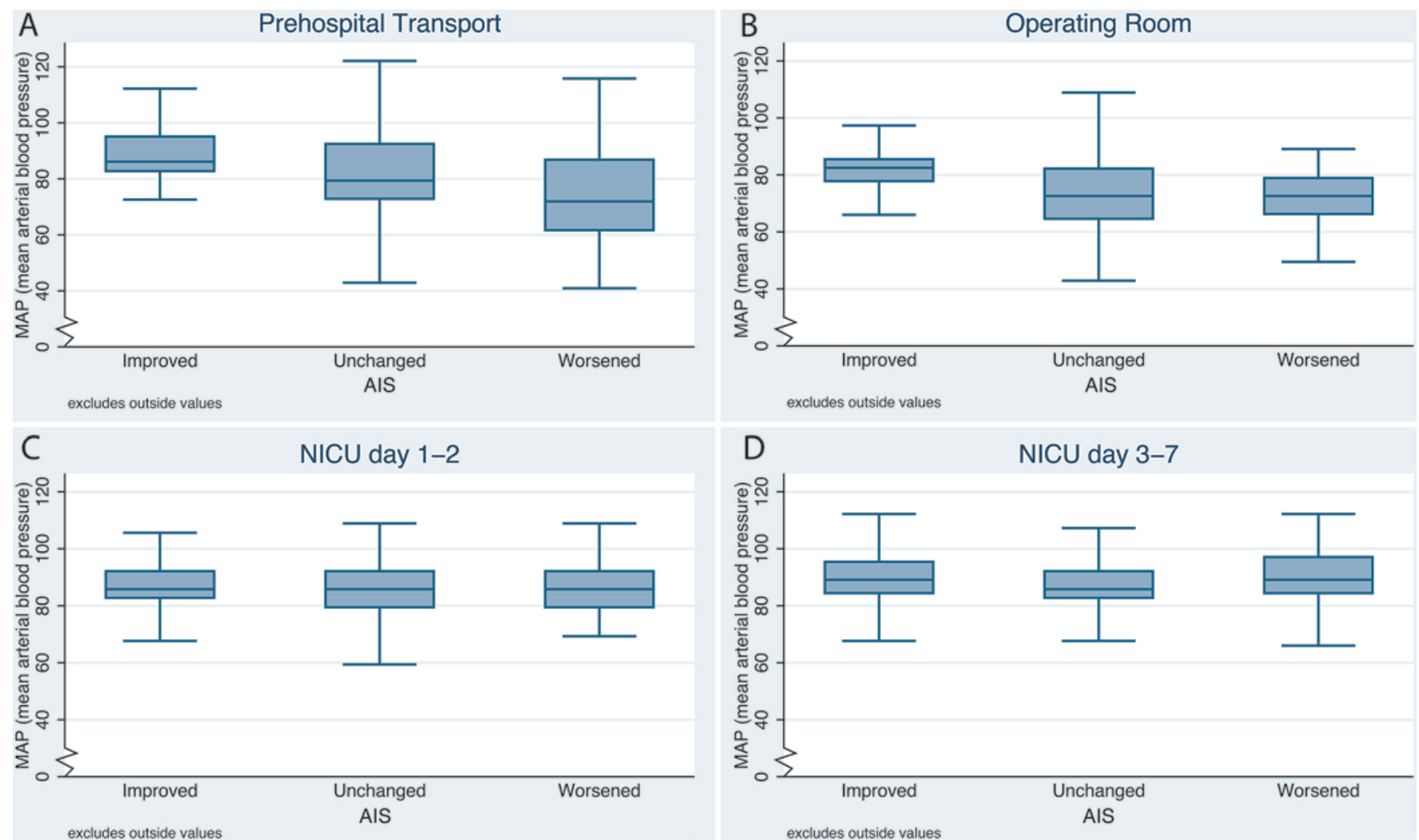

FIG. 1. Graphs showing the 25th, 50th (median), and 75th percentiles for prehospital transport, operating room, NICU days 1-2, and NICU days 3-7. Figure is available in color online only.

neurological outcome. ${ }^{6,17,18}$ Continuous sufficient levels of MABP with none or a few event failures correlated with

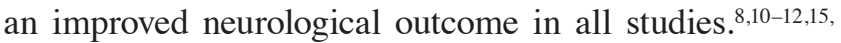
${ }_{17,18}$ The highest MABP values also correlated with the greatest degree of neurological recovery. ${ }^{8,10-12,15,17,18}$ These results are consistent with our findings; however, contrary to earlier findings, our data found no correlation between MABP and the last 5 days in the NICU. This may be due to a well-established intensive care treatment at this time.

We found no earlier studies evaluating the impact of MABP on SCI during transport to the hospital or in the OR. Our study shows a significant impact of MABP $\geq$ $80 \mathrm{~mm} \mathrm{Hg}$ at these two stages. This underlines that it is necessary to put special focus on avoiding hypotension as early as possible in the initial, critical acute phase. Ideally, the treatment target for the SCI should be to ensure optimal perfusion pressure to the injured spinal cord by keeping MABP "high;" however, this treatment approach would conflict with the trauma guideline dogmas that ensure stable circulation before specific organ consideration.

Our study is based on noncontinuous data. During the prehospital transport and in the OR, data are collected every 15 minutes. Data in the NICU are registered every hour for most patients. The staff is instructed to be aware of a significant increase or decrease in MABP in between measurements and to register events when they occur between standard time points. MABP data are collected continuously through invasive blood pressure monitoring at this phase. MABP is a standardized measurement and is considered a measure for organ perfusion pressure.,14 However, MABP is not adjusted in correlation to the intradural pressure, and, thus, the perfusion pressure needed for sufficient perfusion was not known in our cases. ${ }^{1,14}$

Our data were obtained retrospectively and therefore are liable to interpretation bias. Causality between MABP and SCI cannot be established in this study. Patients with a severe trauma could have had a worse outcome due to the primary trauma impact. Furthermore, a higher degree of completeness is more likely to produce neurogenic shock and hence the patient would be at a higher risk of neurological deterioration than less-completely injured patients. $^{9-12}$ This is especially interesting in the prehospital transport where no inotropes are administrated to regulate MABP. However, when the current findings are combined with earlier prospective findings similar to ours, we believe it increases the case for causality.

AIS grades were defined at admission to the hospital. The study premise was that the admission AIS grade is similar to the true initial AIS grade just after the traumatic event. We believe this to be true; however, we cannot rule out divergence. The collected data were not complete for all calculations. This is very hard to avoid in a retrospective study. Bias cannot be excluded; however, the direction of bias is less clear.

Our data suggest that the sufficient management of MABP in SCI patients should be initialized as soon as 
possible. It is, however, a complex matter to increase MABP to levels suggested in traumatic SCI before a final diagnosis is established and other trauma-related events (e.g., internal hemorrhage) are excluded. This is especially true for prehospital treatment and transport. As recommended in the 2013 AANS guidelines, we suggest putting special focus on avoiding hypotension until hospital admission whenever a traumatic SCI is suspected..$^{10}$

\section{Conclusions}

Continuous MABP $\geq 80 \mathrm{~mm} \mathrm{Hg}$ has a significant correlation with and a moderate impact on long-term neurological outcome in the initial critical acute phase after SCI through to the first few days in the NICU. For the remaining period of hospitalization after the first few days, maintaining MABP above this threshold does not seem to correlate with the neurological outcome. When a patient is thought to have sustained an SCI, avoidance of hypotension until final diagnoses are established seems reasonable, and after diagnosis maintaining an MABP > $80 \mathrm{~mm} \mathrm{Hg}$ is encouraged as soon as possible and through the acute SCI phase and in the first few days following in the NICU.

\section{References}

1. Fawcett JW, Curt A, Steeves JD, et al. Guidelines for the conduct of clinical trials for spinal cord injury as developed by the ICCP panel: spontaneous recovery after spinal cord injury and statistical power needed for therapeutic clinical trials. Spinal Cord. 2007;45(3):190-205.

2. Hicken BL, Putzke JD, Richards JS. Bladder management and quality of life after spinal cord injury. Am J Phys Med Rehabil. 2001;80(12):916-922.

3. Krogh K, Nielsen J, Djurhuus JC, et al. Colorectal function in patients with spinal cord lesions. Dis Colon Rectum. 1997;40(10):1233-1239.

4. Kivisild A, Sabre L, Tomberg T, et al. Health-related quality of life in patients with traumatic spinal cord injury in Estonia. Spinal Cord. 2014;52(7):570-575.

5. Levi L, Wolf A, Belzberg H. Hemodynamic parameters in patients with acute cervical cord trauma: description, intervention, and prediction of outcome. Neurosurgery. 1993;33(6):1007-1017.

6. Tee JW, Altaf F, Belanger L, et al. Mean arterial blood pressure management of acute traumatic spinal cord injured patients during the pre-hospital and early admission period. $J$ Neurotrauma. 2017;34(6):1271-1277.

7. Tator $\mathrm{CH}$, Fehlings MG. Review of the secondary injury theory of acute spinal cord trauma with emphasis on vascular mechanisms. J Neurosurg. 1991;75(1):15-26.

8. Piepmeier JM, Lehmann KB, Lane JG. Cardiovascular instability following acute cervical spinal cord trauma. Cent Nerv Syst Trauma. 1985;2(3):153-160.

9. Burns S, Biering-Sørensen F, Donovan W, et al. International standards for neurological classification of spinal cord injury, revised 2011. Top Spinal Cord Inj Rehabil. 2012;18(1):85-99.

10. Ryken TC, Hurlbert RJ, Hadley MN, et al. The acute cardiopulmonary management of patients with cervical spinal cord injuries. Neurosurgery. 2013;72(suppl 2):84-92.
11. Summers RL, Baker SD, Sterling SA, et al. Characterization of the spectrum of hemodynamic profiles in trauma patients with acute neurogenic shock. J Crit Care. 2013;28(4):531. e1-531.e5.

12. Saadoun S, Chen S, Papadopoulos MC. Intraspinal pressure and spinal cord perfusion pressure predict neurological outcome after traumatic spinal cord injury. J Neurol Neurosurg Psychiatry. 2017;88(5):452-453.

13. Vale FL, Burns J, Jackson AB, Hadley MN. Combined medical and surgical treatment after acute spinal cord injury: results of a prospective pilot study to assess the merits of aggressive medical resuscitation and blood pressure management. J Neurosurg. 1997;87(2):239-246.

14. Casha S, Christie S. A systematic review of intensive cardiopulmonary management after spinal cord injury. $\mathrm{J} \mathrm{Neu}$ rotrauma. 2011;28(8):1479-1495.

15. Hadley MN, Walters BC, Grabb PA, et al. Guidelines for the management of acute cervical spine and spinal cord injuries. Clin Neurosurg. 2002;49:407-498.

16. Walters BC, Hadley MN, Hurlbert RJ, et al. Guidelines for the management of acute cervical spine and spinal cord injuries: 2013 update. Neurosurgery. 2013;60(CN_suppl_1):82-91.

17. Dakson A, Brandman D, Thibault-Halman G, Christie SD. Optimization of the mean arterial pressure and timing of surgical decompression in traumatic spinal cord injury: a retrospective study. Spinal Cord. 2017;55(11):1033-1038.

18. Hawryluk G, Whetstone W, Saigal R, et al. Mean arterial blood pressure correlates with neurological recovery after human spinal cord injury: analysis of high frequency physiologic data. J Neurotrauma. 2015;32(24):1958-1967.

\section{Disclosures}

The authors report no conflict of interest concerning the materials or methods used in this study or the findings specified in this paper.

\section{Author Contributions}

Conception and design: Haldrup, Rasmussen. Acquisition of data: Haldrup. Analysis and interpretation of data: Haldrup, Rasmussen. Drafting the article: Haldrup, Rasmussen. Critically revising the article: Haldrup, Thygesen, Kirkegaard, Kasch, Rasmussen. Reviewed submitted version of manuscript: all authors. Approved the final version of the manuscript on behalf of all authors: Haldrup. Statistical analysis: Dyrskog. Administrative/technical/material support: all authors. Study supervision: Rasmussen.

\section{Supplemental Information \\ Previous Presentations}

Portions of this paper were presented in oral form at EUROSPINE 2019, in Helsinki, Finland, October 18, 2019, and at the 2nd Nordic Neurotrauma Conference, in Lund, Sweden, November 20, 2019.

\section{Correspondence}

Mette Haldrup: Aarhus University Hospital, Aarhus, Denmark. methalje@rm.dk. 